\title{
Die Figurativität der allgemeinen Wissenschaftssprache des Deutschen
}

\author{
Markus Rheindorf (Wien)
}

\begin{abstract}
Academic discourse relies on a common register that reaches across disciplinary boundaries. This so-called common language of academia includes terms denoting common objects and actions of academic work in the fields of knowing, understanding and learning, of perceiving and observing, of designing and devising as well as of writing and speaking in their many different forms. In German academic discourse, this common register is highly figurative, often creating problems in bilingual contexts as well as during students' acquisition of academic discourse. This paper presents an ongoing project to identify, describe and categorize the lexical dimension of this common language of academia by means of corpuslinguistic analyses. It presents operators to distinguish said register from non-academic German as well as discipline-specific discourse. Following these filtering procedures, the figurativity of the identified items is discussed in terms of their spatial-haptic, optical-visual, mental-cognitive and acoustic-linguistic character. Prominent cases of ambiguity, when specific lexical items can be used and read as expressing two figurative aspects, are also identified.
\end{abstract}

\section{$1 \quad$ Einleitung}

So wie die spezifische Fachsprache der einzelnen Disziplinen oft ein trennendes Element in der interdisziplinären Forschung und Kommunikation ist, so ist die allgemeine Wissenschaftssprache (AWS) ein verbindendes Element. Die Linguistin arbeitet einen Punkt heraus, ebenso wie ein Physiker. Historiker und Juristin argumentieren, stellen dar, stellen gegenüber und diskutieren in ihren Publikationen. Sie alle bedienen sich eines Grundstocks an Wörtern, der in allen Wissenschaften im deutschen Sprachraum vergleichbar verwendet wird und sich in dieser Verwendung zugleich von der Alltagssprache unterscheidet. Dank dieser allgemeinen Wissenschaftssprache können sie über wesentliche Prozeduren und Handlungen der Wissenschaft miteinander kommunizieren.

Dem Begriff der ,allgemeinen Wissenschaftssprache“ liegt die Beobachtung zugrunde, dass die Wissenschaftssprache nicht nur aus Fachsprache besteht, wenngleich letztere ein essentieller Bestandteil dieser ist. Im Gegensatz zur disziplinengebundenen Terminologie der einzelnen Fächer und Wissenschaften schließt der allgemeine wissenschaftliche Wortschatz nach Schepping (1976) kontinuierlich an den in Schule, Hochschule und Berufsausbildung vermittelten „gehobenen Wortschatz“ an: $\mathrm{Er}$ ist im Unterschied zur speziellen 
terminologischen Lexik der einzelnen Disziplinen überschaubar, steht jenseits fachlicher und wissenschaftlicher Bezeichnungsinnovation (etwa für neue Sachverhalte), ist an keine spezielle Thematik gebunden und zeigt eine große Wiederholungsrate. Die AWS stellt somit das lexikalische Grundinventar der wissenschaftlichen und wissenschaftsvermittelnden Kommunikation $\mathrm{zu}$ Verfügung und unterscheidet sich damit von Fachsprache (vgl. Strauß/Zifonun 1985: 84). Auf Basis von Erks (1972) Untersuchungen zu den lexikalischen Elementen wissenschaftlicher Fachtexte sieht Schepping (1976) die AWS als einen begrenzten Bestand feststehender Topoi und wiederkehrender Wendungen, die als notwendiges Fundament für die Spezialterminologien der Fächer fungieren. Mit ihren „mannigfaltigen Ausdrucksformen für die Gesten des Voraussetzens, des Begründens, des Folgerns, des Ableitens, des Einschränkens, des Übertragens und Vergleichens, des Benennens und Bestimmens“ (Schepping 1976: 21) bildet sie ein allgemeines Instrumentarium zur Gestaltung und Kommunikation von Wissen.

Mit Blick auf eine Wissenschaftssprachkomparatistik definiert Ehlich (1993) die AWS als jene „fundamentalen sprachlichen Mittel [...], derer sich die meisten Wissenschaften gleich oder ähnlich bedienen“ (33). Er betont, dass die AWS Wissensbestände zueinander, zur erfahrbaren Wirklichkeit, aber auch zur Wissenschaft als Prozess und Verfahren in Beziehung setzt (vgl. Ehlich 1998: 856-858, 1999: 9-11). Zu diesem Zweck habe sich, so Ehlich (1993) in diesem Zusammenhang weiter, im Deutschen ein an die Alltags- oder Gemeinsprache angelehntes Vokabular herausgebildet, das nicht fachspezifisch sondern fächerübergreifend verwendet wird, das aber gegenüber dem gemeinsprachlichen Gebrauch eine Bedeutungswandlung durchgemacht hat. Die AWS kann letztlich nicht als domänenspezifisch (also wissenschaftsspezifisch) im engen Sinn gesehen werden, da zahlreiche Wörter auch in anderen Domänen (etwa Journalistik) vorkommen, wenn auch häufig in anderer Bedeutung oder Kollokation. Der Status der AWS lässt sich daher konzeptuell durch den Begriff der „Typik“ weit besser fassen als durch den (ausschließenden) Begriff der „Domänenspezifik“ (vgl. Feilke/Steinhoff 2003; Feilke 2010). Die Lexik der AWS, aber auch ihre präferierten Kollokationen, sind typisch anstatt spezifisch, d. h. besonders charakteristisch aber nicht ausschließlich.

\section{Figurativität in den Wissenschaften}

Die AWS des Deutschen beinhaltet viele Begriffe, die der alltäglichen Sprache entlehnt wurden und nunmehr eine abgewandelte, metaphorische Bedeutung haben - wie etwa die Verben heranziehen, herausarbeiten oder beleuchten. Die darin begründete Figurativität der allgemeinen deutschen Wissenschaftssprache ist zugleich sprach- und zum Teil auch kulturspezifisch, was der Versuch einer Übersetzung in andere europäische Wissenschaftssprachen, etwa der Englischen, deutlich macht. Im englischen Kulturraum haben sich andere Begriffsfelder für den metaphorischen Gebrauch in der Wissenschaftssprache herauskristallisiert. Herausarbeiten ist auch hierfür ein gutes Beispiel: Der Vergleich mit dem Englischen work out zeigt deutlich, dass sich nicht notwendigerweise auch in sprachgeschichtlich verwandten Sprachen eine wissenschaftsspezifische Bedeutung herausbildet, denn work out weist nicht nur nicht dieselbe Bedeutung, sondern keinerlei wissenschaftsspezifische Bedeutung auf. Eine mögliche und für die internationale wissenschaftliche Arbeit höchst relevante Erklärung solcher Unterschiede in den jeweiligen 
AWS bieten Fandrych (2002) und Ehlich (1995). Sie argumentieren, dass sich in der AWS „zentrale Konzeptualisierungen des wissenschaftlichen Sprachhandelns und des Forschungsund Erkenntnisprozesses“ (Fandrych 2002: 1) bzw. Strategien der Erkenntnisgewinnung verschiedener Wissenschaftsepochen aufspüren lassen (vgl. Ehlich 1995).

Von Bedeutung in der oben ausgeführten und im Weiteren korpuslinguistisch präzisierten Definition der AWS ist die Beschränkung auf Aspekte der Lexik einer Wissenschaftssprache. Linguistische Arbeiten zur AWS beschäftigen sich vorwiegend mit lexikalischen Fragen (vgl. u. a. Ehlich 1995, 1999, 2000; Graefen 1999, 2000). Genauer betrachtet lässt sich dieser Fokus noch näher bestimmen: Es wurden vor allem die Verben der AWS des Deutschen im Hinblick auf ihre Figurativität untersucht. Dies liegt zum einen daran, dass beim Erwerb von wissenschaftlicher Schreibkompetenz in diesem Bereich die größten Probleme auftreten und zwar für Sprecher des Deutschen als Erst- und als Zweitsprache. Zum anderen liegt es aber auch daran, dass das Lexikon der AWS von Verben, genauer gesagt von bildlich gebrauchten Sprechhandlungsverben und performativen Verben bzw. ihren Nominalisierung dominiert wird (vgl. Graefen 1997; Hund 1999; Fandrych 2004). Eine umfassende Perspektive muss jedoch alle Wortarten mit einbeziehen; für Nomen (besonders Komposita) und Adjektive wurde dies auch bereits angedacht (vgl. u. a. Graefen 2009).

\section{Die ,allgemeine“ oder „alltägliche“ Wissenschaftssprache des Deutschen}

Um dem Umstand, dass die AWS des Deutschen stark an die Alltagssprache angelehnt ist, konzeptuell Rechnung zu tragen, schlägt Ehlich (1999) an anderer Stelle den Terminus „alltägliche Wissenschaftssprache“ vor. Da sich die metaphorisch überformte Lexik, insbesondere in Form von Komposita wie Forschungslandschaft, nicht auf Alltagsbezüge beschränkt, wäre eine strenge Ausgrenzung aller Begriffe ohne Alltagsbezug jedoch dem Erkenntnisinteresse der Untersuchung der Figurativität der allgemeinen deutschen Wissenschaftssprache entgegengesetzt. Ein weiterer Grund für die hier eingenommene Perspektive auf die AWS als Gesamtheit - ohne Fokus auf das alltägliche Element - liegt in der mittlerweile für Verben erprobten Vorgehensweise, die relevanten Begriffe nicht isoliert, sondern als in semantisch-funktionale Felder eingebettet zu betrachten (vgl. Fandrych 2004; Meißner 2009). Der Blick auf diese Felder ermöglicht zugleich eine Einordnung der figurativen wie auch der funktionalen Aspekte im wissenschaftlichen Gebrauch.

Bisherige Untersuchungen weisen darauf hin, dass bei der Herausbildung der deutschen allgemeinen Wissenschaftssprache vorwiegend auf gemeinsprachlichen Wortschatz zurückgegriffen wurde, welcher jedoch ausgebaut und angepasst wurde. Dabei wurde zwar ebenfalls auf bestehende lateinische Termini und Ausdrucksweisen zurückgegriffen, diese blieben aber stark beschränkt. Sprach- und wissenschaftshistorisch markieren die Protagonisten dieser Anstrengungen (Giordano Bruno, Martin Luther, Leibniz und Thomasius) „Wendepunkte hin zur jeweiligen ,vernakulären“ Sprache“ (Ehlich 2000: 15). 


\section{$4 \quad$ Datengrundlage ${ }^{1}$}

Die Daten- und Ergebnislage zur deutschen AWS ist bestimmt von fragmentarischen Perspektiven und Einzelanalysen. Die linguistischen Arbeiten Ehlichs (1995, 1998, 1999, 2000, 2003), Fandrychs (2002, 2004), Graefens (1999, 2000, 2009) und zahlreicher anderer (z. B. Meißner 2009) bilden einerseits eine wertvolle Grundlage für eine umfassende Analyse ihrer figurativen Elemente, andererseits handelt es sich dabei um durchwegs kleinere Untersuchungen, die sich nicht nur auf einige Verben bzw. Spezialbereiche wie redeberichtende Verben beschränken, sondern auch mit vergleichsweise kleinen Korpora arbeiten. ${ }^{2}$ Obwohl hier wertvolle Einsichten gewonnen wurden, blieb doch der behandelte Ausschnitt der AWS immer stark beschränkt. Wie Ehlich (2000) festhält, bedarf es für die detaillierte Analyse des komplexen Gegenstandes allerdings einer „breit angelegten, begriffsgeleiteten Empirie“ (18). Anders als bei früheren Studien wird für diesen Beitrag ein großes Korpus herangezogen (siehe unten).

Zunächst stellt sich jedoch die Frage, wie das Konzept der AWS für eine solche Studie zu operationalisieren ist. In oben genannten Arbeiten wurde die Zuordnung von Ausdrücken und Wendungen zur AWS häufig nach „muttersprachlicher Intuition“ im Sinne von Introspektion vorgenommen (vgl. Meißner 2009: 33). Das zentrale Bestimmungskriterium der AWS ist die fachübergreifende Verwendung der zugehörigen Ausdrücke (Ehlich 1993: 33). Dieses Merkmal ist korpuslinguistisch in zweifacher Hinsicht $\mathrm{zu}$ operationalisieren: (1) das Vorkommen in allen unterscheidbaren Fachbereichen und (2) die relative Gleichverteilung der Häufigkeiten über die Fachbereiche.

Um den Wortschatz der AWS gemäß seiner Definition als „in Texten zahlreicher Fachrichtungen mit vergleichbaren Frequenzzahlen vertreten“" (Schepping 1976: 22) im Sinne eines Inventars erfassen zu können, wird mit dem Korpus der Berlin-Brandenburgischen Akademie der Wissenschaften ein großes und öffentlich zugängliches Korpus genutzt. ${ }^{3}$ Es ist dies die einzige öffentlich zugängliche Datensammlung deutschsprachiger fachwissenschaftlicher Texte, die korpuslinguistisch aufbereitet ist. Die aktuell umfassten 24.371.647 Token im akademischen Teilkorpus verteilen sich auf unterschiedlichste wissenschaftliche Fachbereiche. Das Korpus eignet sich nicht nur aufgrund seiner Größe und Zugänglichkeit, sondern erlaubt weiters die Differenzierung zwischen Fachbereichen, die Abfrage von Kollokationen, die Suche nach Wortarten sowie die statistische Aufbereitung von Häufigkeiten hinsichtlich ihrer Signifikanz.

Zunächst lassen sich damit die Untersuchungen von Erk (1972) und Schepping (1976) erneuern und erweitern bzw. verfeinern; gleichzeitig wird damit eine Datengrundlage für die Analyse der figurativen Elemente der AWS geschaffen. Die korpuslinguistische Inventarisierung erfolgt in fünf Schritten:

1. Erhebung der häufigsten Lexeme nach Wortarten für Nomen, Verben und Adjektive (einschließlich Adverbien, adjektivisch verwendete Verbformen)

\footnotetext{
${ }^{1}$ Die folgend dargestellte Erhebung eines Inventars der allgemeinen deutschen Wissenschaftssprache wurde von der Universität Wien gefördert und gemeinsam mit Birgit Huemer durchgeführt.

${ }^{2}$ Im Falle von Fandrych (2002) handelt es sich etwa 17 englische und 19 deutsche wissenschaftliche Artikel.

${ }^{3}$ Das Korpus ist mit gewissen Einschränkungen online zugänglich über „DWDS - Das Digitale Wörterbuch der deutschen Sprache des 20. Jahrhunderts“" (www.dwds.de).
} 
2. Filtern der Funktionswörter

3. Filtern der Fachsprache

4. Filtern der Alltagssprache

5. Bestimmung wissenschaftssprachlicher Verben, Nomen und Adjektive (Lemma)

Für diese Schritte werden jeweils spezifische Abfrage- und Filterroutinen verwendet. Die Erhebung der häufigsten Lexeme erfolgte anhand des Korpus und mit Unterstützung der Berlin-Brandenburgischen Akademie der Wissenschaften, die das Korpus betreut. In diesem ersten Schritt wurden alle Lexeme erfasst, die häufiger als 1 Mal pro Million Token im Korpus auftreten. Das anschließende Filtern von Funktionswörtern wie z. B. sein, haben und Pronomen wie es, er, sie oder wir war einfach umzusetzen, denn sie sind klar definiert und in ihrer Anzahl klar begrenzt. Sie treten in anderen Sprachbereichen ebenso auf wie in der Wissenschaftssprache und haben keine besondere Verwendungsweise oder Bedeutung in der Wissenschaft entwickelt.

Wesentlich aufwendiger ist das Filtern von Fachsprache. Fachspezifische Begriffe oder Termini sind nicht Teil der allgemeinen Wissenschaftssprache. Die Forschung hat diese Abgrenzung zwar theoretisch-konzeptuell und sprachhistorisch thematisiert (Fandrych 2002; Feilke 2010; Graefen 2009; Kretzenbacher/Weinrich 1995), methodisch aber nur unzureichend operationalisiert bzw. rein intuitiv gehandhabt. In der korpusbasierten Frequenzliste finden sich etliche Ausdrücke der verschiedenen (u. a. juristischen, soziologischen) Fachsprache. Die Entscheidung, ob ein Wort ins Inventar der AWS aufzunehmen ist oder nicht, kann über den Vergleich der Teilwerte in der Häufigkeitsverteilung der einzelnen Fachbereiche getroffen werden, für den statistische Operatoren herangezogen werden (unterschieden werden Geisteswissenschaften, Sozialwissenschaften, Technische Wissenschaften, Naturwissenschaften und Angewandte Wissenschaften). Als statistische Indikatoren fungieren dabei Mittel und Median in Relation zueinander (Mittelabweichung). Wird der Grenzwert 1 in dieser Relation überschritten, ist die gleiche oder ähnliche Verteilung in allen Fachbereichen (im Sinne der Definition) fragwürdig.

In der Praxis erweist sich dieser erste Indikator zwar grundsätzlich als robust, aufgrund der mathematischen Eigenschaften der Operatoren überschreiten allerdings auch einige wenige Wörter mit ähnlicher Häufigkeit den Wert 1 geringfügig. Für diese Fälle ist ein zweiter Indikator notwendig: Wenn nicht mehr als eine der fünf wissenschaftlichen Fachgruppen den Mittelwert der anderen Fachbereiche signifikant unter- oder überschreitet, ist das Wort der AWS zuzurechnen.

Um die oben definierte Typik der AWS im Sinne einer Abgrenzung von der Alltagssprache statistisch zu operationalisieren, sind ähnlich wie bei der Fachsprache konkrete Operatoren notwendig. Eine Möglichkeit hierfür bietet der Vergleich zwischen Wissenschaftskorpus und Gesamtkorpus (Zeitungen, Belletristik und Gebrauchsliteratur). Als Beleg für die wissenschaftstypische Verwendung gilt, wenn das Wort in Wissenschaftstexten deutlich häufiger vorkommt als im Restkorpus. Zusätzlich wird über Kollokationen die typische Verwendung überprüft. 
Eine eindeutige Abweichung in der Frequenz ergibt sich etwa bei Tag, das in nichtwissenschaftlichen Texten mehr als doppelt so häufig vorkommt, wie in wissenschaftlichen, und daher nicht als wissenschaftstypisch gelten kann.

\begin{tabular}{|l|l|l|l|}
\hline Tag/e & Token & per Mio. & Korpus Token \\
\hline wissenschaftliche Texte & 7.544 & 309,54 & 24.371 .647 \\
\hline nicht-wiss. Texte & 65.053 & 660,81 & 98.444 .363 \\
\hline
\end{tabular}

Tabelle 1: Abgrenzung von Alltagssprache anhand von Frequenzen

Weniger eindeutig stellt sich die Verteilung bei Frage dar. Im Fall einer solchen geringfügigen Abweichung bzw. nahezu Gleichverteilung muss die zweite Routine, d. h. der Vergleich der Kollokationen in wissenschaftlichen und nicht-wissenschaftlichen Texten, hinzugezogen werden.

\begin{tabular}{|l|l|l|l|}
\hline Frage/n & Token & per Mio. & Korpus Token \\
\hline wissenschaftliche Texte & 13.432 & 551,15 & 24.371 .647 \\
\hline nicht-wiss. Texte & 49.157 & 499,34 & 98.444 .363 \\
\hline
\end{tabular}

Tabelle 2: Abgrenzung von Alltagssprache anhand von Frequenzen

Weichen hierbei die häufigsten Kollokate deutlich voneinander ab, d. h. sind mehr als $30 \%$ unterschiedlich, liegt eine wissenschaftstypische Verwendung des Wortes (im Sinne der Definition) vor. Für Frage ergibt sich demnach eine wissenschaftstypische Verwendung, da sich 46,67\% der Kollokate unterscheiden.

\begin{tabular}{|c|c|c|c|}
\hline $\begin{array}{l}\text { wiss. Kollokate von } \\
\text { Frage }\end{array}$ & $\begin{array}{l}\text { Frequenz des Bigrams } \\
\text { (per Mio.) }\end{array}$ & $\begin{array}{l}\text { nicht-wiss. Kollokate } \\
\text { von Frage }\end{array}$ & $\begin{array}{l}\text { Frequenz des Bigrams } \\
\text { (per Mio.) }\end{array}$ \\
\hline 1. wichtige & $118(4,84)$ & 1. deutsche & $1412(14,34)$ \\
\hline 2. andere & $104(4,27)$ & 2. wichtige & $975(9,90)$ \\
\hline 3. entscheidende & $62(2,54)$ & 3. solche & $808(8,21)$ \\
\hline 4. politisch & $61(2,50)$ & 4. politische & $765(7,77)$ \\
\hline 5. Römische & $54(2,22)$ & 5. ganze & $750(7,62)$ \\
\hline 6. schwierige & $51(2,09)$ & 6. internationale & $688(6,99)$ \\
\hline 7. erste & $50(2,05)$ & 7. unsere & $673(6,84)$ \\
\hline 8. praktische & $46(1,89)$ & 8. große & $653(6,63)$ \\
\hline 9. letzte & $45(1,85)$ & 9. beide & $625(6,35)$ \\
\hline 10. philosophische & $44(1,81)$ & 10. kommende & $513(5,21)$ \\
\hline 11. soziale & $44(1,81)$ & 11. offene & $505(5,13)$ \\
\hline 12. offene & $41(1,68)$ & 12. soziale & $499(5,07)$ \\
\hline 13. gestellte & $40(1,64)$ & 13. neue & $483(4,91)$ \\
\hline 14. große & $40(1,64)$ & 14. folgende & $482(4,90)$ \\
\hline 15. grundsätzliche & $40(1,64)$ & 15. wirtschaftliche & $459(4,66)$ \\
\hline
\end{tabular}




\begin{tabular}{|l|l|l|l|}
\hline 16. weitere & $39(1,60)$ & 16. allgemeine & $429(4,36)$ \\
\hline 17. aufgeworfene & $38(1,56)$ & 17. europäische & $395(4,01)$ \\
\hline 18. deutsche & $38(1,56)$ & 18. letzte & $380(3,86)$ \\
\hline 19. allgemeine & $35(1,44)$ & 19. verschiedene & $324(3,29)$ \\
\hline 20. zweite & $34(1,40)$ & 20. grundsätzliche & $317(3,22)$ \\
\hline 21. solche & $32(1,31)$ & 21. nationale & $314(3,19)$ \\
\hline 22. neue & $31(1,27)$ & 22. entscheidende & $288(2,93)$ \\
\hline 23. folgende & $30(1,23)$ & 23. gute & $274(2,78)$ \\
\hline 24. religiöse & $30(1,23)$ & 24. besondere & $269(2,73)$ \\
\hline 25. umstrittene & $30(1,23)$ & 25. mögliche & $265(2,69)$ \\
\hline 26. ganze & $28(1,15)$ & 26. aktuelle & $264(2,68)$ \\
\hline 27. logische & $28(1,15)$ & 27. wesentliche & $260(2,64)$ \\
\hline 28. prinzipielle & $21(0,86)$ & 28. eigentliche & $249(2,53)$ \\
\hline 29. empirische & $19(0,78)$ & 29. bestimmte & $236(2,40)$ \\
\hline 30. nationale & $19(0,78)$ & 30. schwierige & $235(2,39)$ \\
\hline 31. strittige & $18(0,74)$ & 31. praktische & $204(2,07)$ \\
\hline
\end{tabular}

Tabelle 3: Abgrenzung von Alltagssprache anhand von Kollokaten

Das mittels der oben dargestellten Schritte reduzierte Inventar der deutschen AWS enthält insgesamt 1701 Wörter, davon 643 Verben, 491 Nomen und 567 Adjektive. Im Fall der Verben der deutschen AWS handelt es sich zu einem guten Teil um Präfixverben, also Verben, die einen eigenständigen Verbstamm haben und mit einem Präfix gebildet werden (etwa zeigen und aufzeigen, denken und andenken, greifen und begreifen). In einigen Fällen hat nur die Präfixvariante dieser Verbvarianten eine wissenschaftstypische Bedeutung. Typischerweise wird durch das Präfix eine Bedeutungsverschiebung oder Konkretisierung bewirkt, in den meisten Fällen aber auch Figurativität hinzugefügt oder bereits vorhandene abgewandelt (wie z. B. begreifen, festhalten oder gegenüberstellen).

Die Nomen der deutschen AWS wurden einschließlich Nominalisierungen von Verben oder Adjektiven erhoben (z. B. Diskussion oder Betrachtung). Auch bei den deutschen Adjektiven und Adverbien stellt sich eine konzeptuelle Frage hinsichtlich adjektivisch verwendeter Verbformen wie Partizipien (z. B. grundlegend oder entgegengesetzt). Einige sind so häufig, dass sie unter den häufigsten Adjektiven zu werten sind. Hier stellt sich in der Analyse die Frage, ob diese Belege unter dem jeweiligen Verbstamm oder unter den Adjektiven zu berücksichtigen sind. Die getrennte Anführung als Adjektiv wurde gewählt, weil die Funktionalität in dieser Form eine besondere ist und in einigen Fällen das Verb nur in Partizipform häufig oder besonders häufig auftritt. 


\section{$5 \quad$ Bisherige Analysen und Kategorien}

Als erster Schritt zur Entwicklung von Analysekategorien für die Figurativität der AWS erscheint es sinnvoll, die teils stark voneinander abweichenden Kategorisierungen bisheriger Analysen zu diskutieren. ${ }^{4}$ In seiner Studie unterschied Schepping (1976: 24-26) zwei in sich weiter differenzierte Gruppen von Begriffen der AWS: 1) logisch-methodologisches Vokabular (logisches Schließen wie folgern; sprachlich-begriffliches Repräsentieren wie darstellen) und 2) strategisch-rhetorisch fungierende Textelemente mit vorwiegend metakommunikativer und metadiskursiver Funktion (Markierung des Anfangs, Übergangs oder Abschlusses wie zusammenfassen; ebenso Aspekte der Argumentation wie zeigen und widersprechen; sowie Markierung der Aufmerksamkeit wie hervorheben und Verweisen wie zufolge). Grundsätzlich entspricht schon Schepping (1976) damit ansatzweise der von Thielmann (2009) formulierten Anforderung, für die Analyse von Wissenschaftssprache müssen sprachliche Mittel auf ihre kommunikativen Zwecke untersucht werden. Thielmann schlägt hierfür ganz konkret die von Ehlich und Rehbein konzipierte Funktionale Pragmatik vor, in der sprachliche Formen auf das sprachliche Handeln selbst bezogen werden. Figurativität selbst muss in diesem Rahmen als funktional verstanden werden.

Ein guter Teil der von Schepping (1976) behandelten Wörter der deutschen AWS sind Verben, ergänzt durch einige Adjektive und Nomina. Bei den Verben handelt es sich vor allem um Sprechhandlungsverben und wissenschaftstypische Handlungsverben. Zwar kommen viele dieser auch mit einer verwandten Bedeutung in der Allgemeinsprache vor, ihre wissenschaftsspezifische Bedeutung hat aber eine figurative Wandlungen oder Überformung erfahren (siehe etwa heranziehen). Bei vielen dieser Verben, wie z. B. zeigen, offenlegen, deuten oder herausarbeiten, gibt es keine primäre Sprechhandlungsbedeutung $-\mathrm{d}$. h. sie bezeichnen in ihrer alltagssprachlichen Verwendung keine Sprechhandlungen. Es liegen also metaphorische Prozesse zugrunde, von denen anzunehmen ist, dass sie auf wichtige Modellvorstellungen von wissenschaftlichem Handeln hinweisen (vgl. Hund 1999: 308-310).

Speziell für diese Handlungsverben der allgemeinen Wissenschaftssprache wurden bereits Kategorisierungen vorgeschlagen, wie z. B. die Handlungstypen „Textkommentierung“ und „Literaturbesprechung“ (Fandrych 2002: 4) oder auch die Handlungskategorien „Bewegung“, „Positionierung“, „Transfer“, „Verbindung“, „Greifen“, „Zeigen“ und „Wahrnehmung“ (Meißner 2009: 101). Während diese Kategorisierungen aus Einzelstudien zwar geeignet sind, um der jeweiligen Fragestellung gemäß spezielle Aspekte deutlich zu machen, sind sie für eine umfassende Studie nicht hinreichend, weil sie nur einen Teilbereich fokussieren.

Für die Erarbeitung eines geeigneten Kategorienrasters möchte ich zwei (bisher nicht umgesetzte) Ergänzungsvorschläge aufgreifen. Dies betrifft zunächst Steinhoffs Hinweis, dass „,begriffliche Analysen allein nicht ausreichen, um die Domänentypik des Sprachgebrauch hinreichend zu beschreiben und zu erklären“" (Steinhoff 2007: 87). Um den domänentypischen Sprachgebrauch zu dokumentieren und etwa zu zeigen, ob ein konkretes Verb in der Funktion der Literaturbesprechung oder der Textkommentierung verwendet wird, bedürfe es einer Analyse der Kollokationen mit korpuslinguistischen Mitteln (ähnlich argumentiert auch

\footnotetext{
${ }^{4}$ Eine zentrale Schwierigkeit bei der Untersuchung der AWS besteht darin, das große Feld von Ausdrücken sinnvoll zu ordnen. Im Laufe der Zeit wurden daher bei dem Versuch, dieses Problem zu lösen, zahlreiche Kategorienraster entwickelt (vgl. Steinhoff 2009: 101).
} 
Fandrych 2002). Die funktionalistische Erklärung allein ist also nicht ausreichend, u.a. wegen des „Formulierungsspielraumes“: die Empirie zeigt, dass bestimmte Kollokationen gegenüber anderen mit äquivalenten Handlungsfunktionen präferiert werden (Steinhoff 2007: 88, siehe auch Rothkegel 1994: 517).

Besonders bei „verfahrensorientierten Wendungen“, die das wissenschaftliche Handeln vergegenständlichen und damit für die AWS von großer Bedeutung sind, sind Kollokationsmuster bestimmend. Schlieben-Lange/Kreuzer (1983: 7) haben darauf hingewiesen, dass „gerade die modernen Wissenschaftssprachen zu einem großen Teil verfahrensorientiert sind, also fast mehr sprachliche Ausdrücke für das Verfahren zur Gewinnung, Überprüfung und Sicherung von Wissen schaffen als für Gegenstände, über die Wissen gewonnen werden soll." $\mathrm{Zu}$ diesem großen Feld wissenschaftssprachlicher Formen zählen z. B. Nomen-Verb-Kollokationen wie einer Frage nachgehen, eine Hypothese vorschlagen, ein Beispiel anführen oder einen Begriff prägen. Weitere Beispiele sind die Formulierungsmuster im Folgenden und etwas festhalten (in allen Varianten von ist festzuhalten bis kann festgehalten werden), die meist an einer Schnittstelle des Textes zur thematischen Organisation und LeserInnenführung verwendet werden (vgl. Rheindorf 2014; Steinhoff 2009: 100). Steinhoff (2009) betont zwei Merkmale dieser Wendungen: ihre Verbindlichkeit und ihre Selektivität. Als drittes Merkmal ist ihre charakteristische Figurativität zu ergänzen.

Unter Berücksichtigung dieser Hinweise erscheint für die Untersuchung der figurativen Elemente der AWS eine Kategorisierung zielführend, die zunächst semantisch-funktionale Felder unterscheidet und sich eng auf die wissenschaftssprachliche Handlungscharakteristik bezieht, um spezifische Routinen des wissenschaftssprachlichen Handelns in den Fokus zu stellen. In Anlehnung an und Erweiterung der Herangehensweise von Fandrych (2002) und Steinhoff (2009) schlage ich folgende Kategorien für die funktionale Ebene der AWS vor:

- Wissenschaftliche Handlung

- Wahrnehmung und Aufmerksamkeit ${ }^{5}$

○ Erkenntnisprozess und Beweisführung

○ Konzeptuelles Forschungshandeln 6

○ Logisches und praktisches Forschungshandeln

- Wissenschaftliche Sprechhandlung

- Beschreiben und Darstellen

○ Erwähnen

\footnotetext{
${ }^{5}$ Diese Kategorie ist insofern zweideutig, als die Verben dieser Kategorie sowohl auf das praktische, mentale oder theoretische Forschungshandeln bezogen werden können als auch auf sprachliche Handlungen in konkreten Texten; sie inkludiert Verben der Fokussierung der Aufmerksamkeit bzw. der aktiven Auswahl und des besonderen Interesses.

6 Die in der Literatur vielfach verwendete Kategorie „räumlich-körperliches“ bzw. „räumlich-haptisches“ Handeln sollte neu gefasst werden, da sie auf alltagssprachlichen Ursprung der Bedeutung fokussiert und nicht die wissenschaftstypische Funktion abbildet. Die meisten dieser Begriffe drücken ein konzeptuelles Forschungshandeln aus; diese Kategorie ergänzt nunmehr die Kategorie „Logisches und praktisches Forschungshandeln". Unstrittig ist die Beobachtung, dass im Deutschen ein Gutteil dieser Verben metaphorisch aus dem Bereich räumlich-haptischen Handelns stammt und diese Bildhaftigkeit konzeptuell von der funktionalen Ebene zu trennen ist.
} 


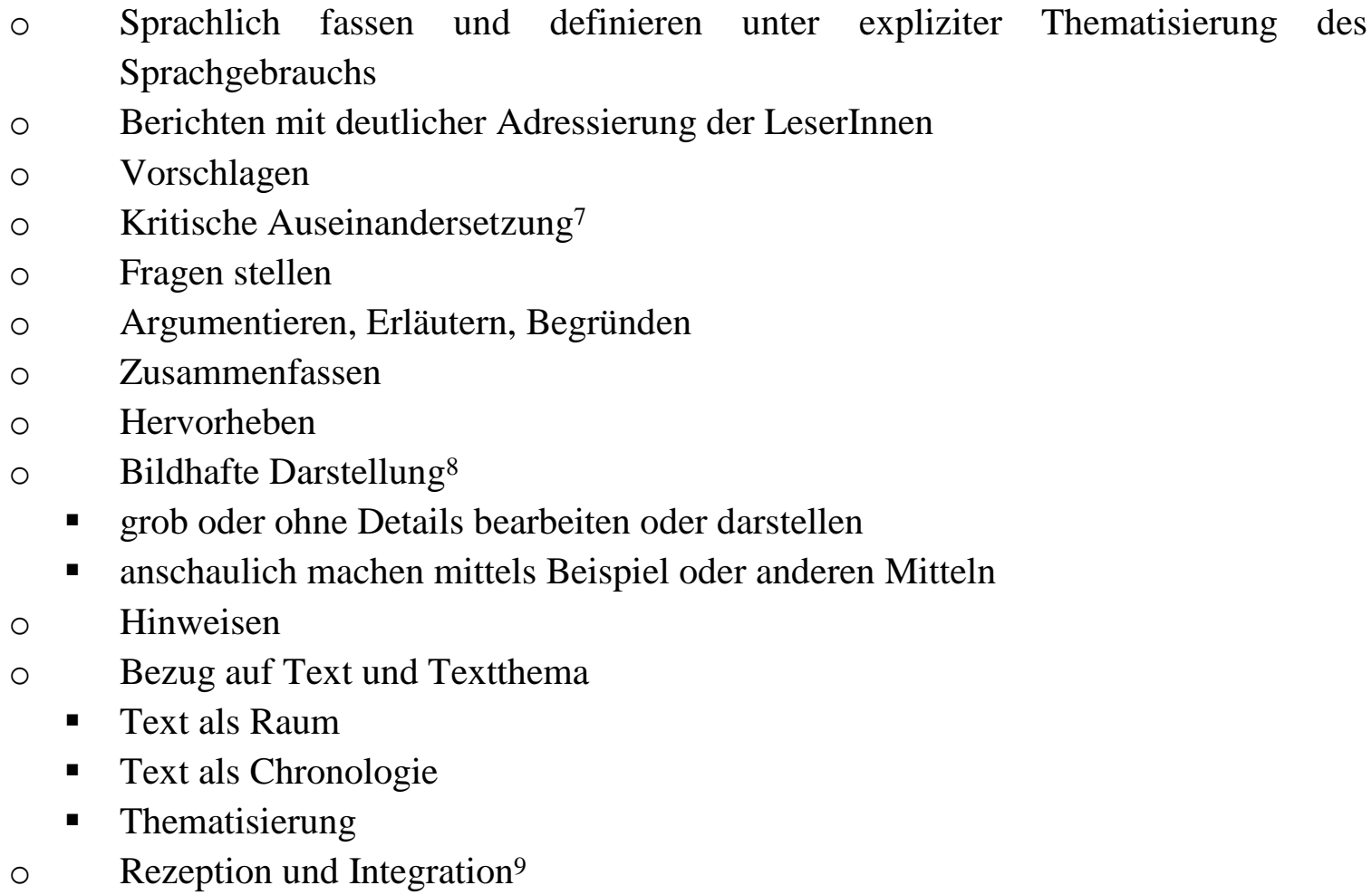

Zweitens müssen die Bildfelder, auf die sich die metaphorische Überformung stützt und aus denen die AWS ein Mehr an Bedeutung bezieht, das über die mit den obigen Kategorien bereits erfassten wissenschaftlichen Handlungen hinausgeht, erfasst werden. Ich schlage folgende Kategorien für die figurative Ebene der AWS vor:

- räumlich-haptisches Bildfeld

- optisch-visuelles Bildfeld

- mental-kognitives Bildfeld

- $\quad$ akustisch-sprachliches Bildfeld

Die Trennung dieser beiden Ebenen hat analytischen Wert und ermöglicht einerseits eine fokussierte Analyse der jeweiligen Ebene und andererseits deren anschließende Zusammenführung. In diesem Beitrag fasse ich die Analyseergebnisse auf der figurativen Ebene der AWS zusammen.

\footnotetext{
${ }^{7}$ Hierzu zählen verschiedene Aspekte des sprachlichen Konflikts um Inhalte, die eine wichtige Rolle bei der Positionierung der eigenen Forschung spielen.

${ }^{8}$ Die in der Literatur gelegentlich verwendete Bezeichnung „Graphische Darstellung“ beschreibt diese Wörter nur ungenau. Neben der veränderten Bezeichnung muss diese Kategorie auch in Form von Unterkategorien präzisiert werden, um die Funktionen der wissenschaftsspezifischen Verwendung besser abzugrenzen.

${ }^{9}$ Diese Kategorie fungiert als annehmendes Gegenstück zur kritischen Auseinandersetzung. Verben dieser Kategorie machen die positive Einschätzung bzw. Übernahme bestimmter Wissenselemente und Positionen anderer deutlich. Fraglich ist, ob rudimentäre Bezüge auf andere nach dem Muster nach Müller oder Müller zufolge erfasst werden sollten. Diese sind relativ häufig und weisen ähnliche Funktionen im Hinblick auf die Akzeptanz, ausstehende Prüfung oder Ablehnung einer Position auf.
} 


\section{Ergebnisse zur Figurativität}

Wendet man das obige Kategorienraster auf das Inventar der deutschen AWS an, zeigen sich folgende Ergebnisse. Insgesamt 433 Wörter der AWS sind figurativ, das sind ca. 25,5\% des Gesamtinventars. Konkret handelt es sich um 245 Verben (38,1\% der Verben), 96 Nomen (19,6\% der Nomen), 92 Adjektive (16,2\% der Adjektive). Der Appendix enthält die vollständigen Listen und Kategorisierung der erfassten Verben, Nomen und Adjektive.

\begin{tabular}{|l|l|l|}
\hline & $\begin{array}{l}\text { Lexeme der AWS mit Häufigkeit } \\
\text { über 1/Mio. Token }\end{array}$ & $\begin{array}{l}\text { Figurative Lexeme der AWS mit } \\
\text { Häufigkeit über 1/Mio. Token }\end{array}$ \\
\hline Verben & 643 & 245 \\
\hline Nomen & 491 & 96 \\
\hline Adjektive & 567 & 92 \\
\hline Gesamt & 1701 & 433 \\
\hline
\end{tabular}

Tabelle 4: Überblick zur Anzahl der figurativen Verben, Nomen und Adjektive in der AWS

Differenziert nach Bildfeldern entfallen insgesamt 321 Wörter auf räumlich-haptische Metaphern (74,2\%), 33 auf optisch-visuelle (7,7\%), ebenfalls 33 auf mental-kognitive $(7,7 \%)$ und 26 auf akustisch-sprachliche (6\%). Hinzu kommen 19 Fälle von Mischformen bzw. Kombinationen aus 2 Bildfeldern (4,4\%). Tabelle 5 gibt die Ergebnisse im Überblick wieder.

\begin{tabular}{|l|l|l|l|l|}
\hline & Verben & Nomen & Adjektive & Gesamt \\
\hline räumlich-haptisch & 186 & 60 & 75 & 321 \\
\hline optisch-visuell & 14 & 12 & 7 & 33 \\
\hline mental-kognitiv & 13 & 14 & 6 & 33 \\
\hline akustisch-sprachlich & 21 & 2 & 3 & 26 \\
\hline Mischformen/Kombinationen & 11 & 7 & 1 & 19 \\
- räumlich-haptisch/mental-kognitiv & 2 & 5 & - & 7 \\
- räumlich-haptisch/akustisch- & 2 & 1 & - & 3 \\
$\quad$ sprachlich & 7 & 1 & 1 & 9 \\
- räumlich-haptisch/optisch-visuell/ & & & & \\
\hline Gesamt & & & & 433 \\
\hline
\end{tabular}

Tabelle 5: Detailergebnisse zur Figurativität der AWS nach Wortart

Ein näherer Blick auf die Ausprägung der Figurativität der einzelnen Wortarten zeigt deutlich, dass für Verben, Nomen und Adjektive gleichermaßen das räumlich-haptische Bildfeld überwiegt. Bei den Verben der AWS entfallen 75,9\% auf räumlich-haptische Bilder, 8,6\% auf akustisch-sprachliche, 5,7\% auf optisch-visuelle und nur 5,3\% auf mental-kognitive. Bei den Mischformen, die alle räumlich-haptische Aspekte enthalten, kommen vor allem optischvisuelle Bilder hinzu. Bei den Nomen der AWS entfallen 62,5\% auf räumlich-haptische Bilder, 14,6\% auf mental-kognitive, 12,5\% auf optisch-visuelle und nur 2,1\% auf akustischsprachliche. Bei den Mischformen, die alle räumlich-haptische Aspekte enthalten, kommen vor allem mental-kognitive Bilder hinzu. Bei Verben der AWS entfallen 81,5\% auf räumlichhaptische Bilder, 7,6\% auf optisch-visuelle, 6,5\% auf mental-kognitive und nur 3,3\% auf akustisch-sprachliche. Bei den Mischformen, die alle räumlich-haptische Aspekte enthalten, kommt ein optisch-visuelles Bild hinzu. Damit bestätigt die detaillierte Analyse den häufig 
geäußerten Eindruck, die deutsche Wissenschaftssprache sei erstens von starker Bildhaftigkeit geprägt und zweitens sei diese vor allem räumlich-haptische Figurativität (Fandrych 2002, 2004; Hund 1999; Meißner 2009).

\section{$7 \quad$ Ausblick}

Auch wenn die funktionale Perspektive auf die allgemeine Wissenschaftssprache in diesem Beitrag nur angerissen werden konnte, zeigen die obigen Ergebnisse und die im Appendix wiedergegebene Kategorisierung des Inventars bereits Muster für die Funktionen „wissenschaftliche Handlung“ und „wissenschaftliche Sprechhandlung“. Aufgrund der funktionalen Ambiguität bzw. kontextabhängigen Flexibilität vieler Ausdrücke der allgemeinen Wissenschaftssprache des Deutschen erfordert eine detaillierte Kategorisierung des Inventars auf der funktionalen Ebene ausgiebigen Raum und detaillierte Darstellung. Die vorwiegend räumlich-haptische Konzeptualisierung des Textraumes, in dem wissenschaftliches Handeln und Erkenntnis dargelegt (sic) werden können, mag als Entsprechung der deutschen Wissenschafts- und Schreibtradition gesehen werden. Sie entfaltet - mitunter breit und mäandernd - einen Referenzraum, der die Forschungslandschaft ebenso miteinschließt wie die Verortung der eigenen Vorgehensweise in ihr. Die evokative, imaginierende Dimension dieser Versprachlichung von Wissenschaft ist gleichermaßen plastisch wie behäbig, hat Ausdehnung, Masse und Schwere.

\section{Literaturverzeichnis}

Ehlich, Konrad (1993): „Deutsch als fremde Wissenschaftssprache“. Jahrbuch Deutsch als Fremdsprache 19: 13-42.

Ehlich, Konrad (1995): „Die Lehre der deutschen Wissenschaftssprache: sprachliche Strukturen, didaktische Desiderate.“ In: Kretzenbacher, Heinz/Weinrich, Harald (eds.): Linguistik der Wissenschaftssprache. Berlin/New York, de Gruyter: 325-352.

Ehlich, Konrad (1998): „Kritik der Wissenschaftssprachen.“ In: Hoffmann, Lothar/ Kalverkämper, Hartwig/Wiegand, Herbert E. (eds.): Fachsprachen. Ein internationales Handbuch. Berlin/ New York, de Gruyter: 856-866.

Ehlich, Konrad (1999): „Alltägliche Wissenschaftssprache.“ InfoDaF 26: 3-24.

Ehlich, Konrad (2000): „Deutsch als Wissenschaftssprache für das 21. Jahrhundert.“ German as a Foreign Language GFL 1/2000. http://www.gfl-journal.de/1-2000/ehlich.html [26.05.2010].

Ehlich, Konrad (2003): „Wissenschaftssprachkomparatistik“. In: Ehlich, Konrad (ed.): Mehrsprachige Wissenschaft - europäische Perspektiven. Eine Konferenz im Europäischen Jahr der Sprachen. München, DaF Institut und: www.euro-sprachenjahr.de.

Erk, Heinrich (1972): Zur Lexik wissenschaftlicher Fachtexte I: Verben - Frequenz und Verwendungsweise. München: Hueber.

Fandrych, Christian (2002): „Herausarbeiten vs. Illustrate: Kontraste bei der Versprachlichung von Sprechhandlungen in der englischen und deutschen Wissenschaftssprache." In: Ehlich, Konrad (ed.): Mehrsprachige Wissenschaft europäische Perspektiven. Eine Konferenz im Europäischen Jahr der Sprachen. München, DaF Institut und: www.euro-sprachenjahr.de [26.05.2010]. 
Fandrych, Christian (2004): „Bilder vom wissenschaftlichen Schreiben. Sprechhandlungsausdrücke im Wissenschaftsdeutschen. Linguistische und didaktische Überlegungen." Materialien Deutsch als Fremdsprache 73: 269-291.

Feilke, Helmuth/Steinhoff, Torsten (2003): „Zur Modellierung der Entwicklung wissenschaftlicher Schreibfähigkeiten." In: Ehlich, Konrad/Steets, Angelika (eds.): Wissenschaftlich schreiben - lehren und lernen. Berlin, de Gruyter: 112-128.

Feilke, Helmuth (2010): „,Aller guten Dinge sind drei ${ }^{\star}$ - Überlegungen zu Textroutinen \& literalen Prozeduren“. In: Fest-Platte für Gerd Fritz. Betreut von Bons, Iris/ Gloning, Thomas/ Kaltwasser, Dennis. www.festschrift-gerd-fritz.de/files/feilke_2010_literaleprozeduren-und-textroutinen.pdf [08.12.2010].

Graefen, Gabriele (1997): „Wissenschaftssprache - eine Thema für den Deutsch-alsFremdsprache-Unterricht?" Materialien Deusch als Fremdsprache 47: 31-44.

Graefen, Gabriele (1999): „Wie formuliert man wissenschaftlich?“ In: Barkowski, Hans/Wolff, Armin (eds.): Alternative Vermittlungsmethoden und Lernformen auf dem Prüfstand. Wissenschaftssprache - Fachsprache; Landeskunde aktuelle; Interkulturelle Begegnungen - Interkulturelles Lernen. Materialien Deutsch als Fremdsprache 52. Regensburg, FaDaF 1999: 222-239.

Graefen, Gabriele (2000): „Einführung in den Gebrauch der Wissenschaftssprache.“ In: Wolff, Armin/Winters-Ohle, Elmar (eds.): Wie schwer ist die deutsche Sprache wirklich? Regensburg, FaDaF 2000: 191-210.

Graefen, Gabriele (2009): „Versteckte Metaphorik - Ein Problem im Umgang mit der fremden deutschen Wissenschaftssprache." In: Dalmas, Martine/Foschi Albert, Marina/Neuland, Eva (eds.): Wissenschaftliche Textsorten im Germanistikstudium deutschitalienisch-französisch kontrastiv. Akten der Trilateralen Forschungskonferenz 2007-2008. Deutsch-Italienisches Zentrum Centro Italo-Tedesco Via Giulio Vigoni: 149-166.

Hund, Eva (1999): „Naheliegend oder weithergeholt - unterrichtspraktische Überlegungen zu Raum und Körper in der Wissenschaftssprache." In: Barkowski, Hans/Wolff, Arnim (eds.): Alternative Vermittlungsmethoden und Lernformen auf dem Prüfstand. Wissenschaftssprache - Fachsprache; Landeskunde aktuelle; Interkulturelle Begegnungen - Interkulturelles Lernen. Materialien Deutsch als Fremdsprache 52. Regensburg, FaDaF 1999: 290-320.

Kretzenbacher, Heinz/Weinrich, Harald (1995): Linguistik der Wissenschaftssprache. Berlin/ New York, de Gruyter: 325-352.

Meißner, Cordula (2009): Figurative Verben in der alltäglichen Wissenschaftssprache des Deutschen. Eine korpuslinguistische Pilotstudie. Centre for Applied Language Studies, University of Jyväskylä.

Rheindorf, Markus (2014): „,zusammenfassend kann festgehalten werden“: Zur Äquivalenz phrasaler Konstruktionen in der deutschen und englischen Wissenschaftssprache aus funktionaler Perspektive“. Wiener Linguistische Gazette 78: 61-82.

Rothkegel, Annely (1994): „Kollokationsbildung und Textbildung.“ In: Sandig, Barbara (ed.): EUROPHRAS 92: Tendenzen der Phraseologieforschung. Studien zur Phraseologie und Parömiologie. Bochum, Universitätsverlag Brockmeyer: 499-523.

Schepping, Heinz (1976): „Bemerkungen zur Didaktik der Fachsprache des Deutschen als Fremdsprache“. In: Rall, Dietrich/Schepping, Heinz/Schleyer, Walter (eds.): Beiträge einer 
Arbeitstagung an der RWTH Aachen vom 30. September bis 4. Oktober 1974. Bonn, DAAD: $13-34$.

Schlieben-Lange, Brigitte/Kreuzer, Helmut (1983): „Probleme und Perspektiven der Fachsprachen- und Fachliteraturforschung." Fachsprache und Fachliteratur 51/52: 7-26.

Steinhoff, Thorsten (2007): Wissenschaftliche Textkompetenz. Sprachgebrauch und Schreibentwicklung in wissenschaftlichen Texten von Studenten und Experten. Tübingen: Niemayer.

Steinhoff, Torsten (2009): „Alltägliche Wissenschaftssprache und wissenschaftliche Textprozeduren. Ein Vorschlag zur kulturvergleichenden Untersuchung wissenschaftlicher Texte.“ In: Dalmas, Martine/Foschi Albert, Marina/Neuland, Eva (eds.): Wissenschaftliche Textsorten im Germanistikstudium deutsch-italienisch-französisch kontrastiv. Akten der Trilateralen Forschungskonferenz 2007-2008. Deutsch-Italienisches Zentrum Centro ItaloTedesco Via Giulio Vigoni: 97-107.

Strauß, Gerhard/Zifonun, Gisela (1985): Die Semantik schwerer Wörter im Deutschen. 1 Lexikologie schwerer Wörter. Tübingen, Narr.

Thielmann, Winfried (1999): „Begründungen versus 'advance organizers' - Zur Problematik des Englischen als lingua franca der Wissenschaft." Deutsche Sprache 4/99: 370-378.

Thielmann, Winfried (2009): „Wissenschaftliches Sprechen und Schreiben an deutschen Universitäten“. In: Dalmas, Martine/Foschi Albert, Marina/Neuland, Eva (eds.): Wissenschaftliche Textsorten im Germanistikstudium deutsch-italienisch-französisch kontrastiv. Akten der Trilateralen Forschungskonferenz 2007-2008. Deutsch-Italienisches Zentrum Centro Italo-Tedesco Via Giulio Vigoni: 47-54.

\section{Anhang}

\begin{tabular}{|l|l|}
\hline Verb & Bildfeld \\
\hline finden & $\begin{array}{l}\text { optisch-visuell/ } \\
\text { räumlich-haptisch }\end{array}$ \\
\hline stehen & räumlich-haptisch \\
\hline liegen & räumlich-haptisch \\
\hline bleiben & räumlich-haptisch \\
\hline gehen & räumlich-haptisch \\
\hline führen & räumlich-haptisch \\
\hline sehen & optisch-visuell \\
\hline sagen & akustisch-sprachlich \\
\hline bestehen & räumlich-haptisch \\
\hline erscheinen & optisch-visuell \\
\hline nennen & akustisch-sprachlich \\
\hline hören & akustisch-sprachlich \\
\hline scheinen & optisch-visuell \\
\hline sprechen & akustisch-sprachlich \\
\hline bezeichnen & räumlich-haptisch \\
\hline wissen & kognitiv-mental \\
\hline enthalten & räumlich-haptisch \\
\hline
\end{tabular}

\begin{tabular}{|l|l|}
\hline darstellen & räumlich-haptisch \\
\hline nehmen & räumlich-haptisch \\
\hline deuten & räumlich-haptisch \\
\hline gelangen & räumlich-haptisch \\
\hline schließen & räumlich-haptisch \\
\hline folgen & räumlich-haptisch \\
\hline suchen & räumlich-haptisch \\
\hline denken & kognitiv-mental \\
\hline verstehen & kognitiv-mental \\
\hline erkennen & kognitiv-mental \\
\hline tragen & räumlich-haptisch \\
\hline erreichen & räumlich-haptisch \\
\hline besitzen & räumlich-haptisch \\
\hline zeigen & $\begin{array}{l}\text { optisch-visuell/ } \\
\text { räumlich-haptisch }\end{array}$ \\
\hline spielen & räumlich-haptisch \\
\hline ziehen & räumlich-haptisch \\
\hline verbinden & räumlich-haptisch \\
\hline fallen & räumlich-haptisch \\
\hline behandeln & räumlich-haptisch \\
\hline
\end{tabular}




\begin{tabular}{|c|c|}
\hline einführen & räumlich-haptisch \\
\hline pflegen & räumlich-haptisch \\
\hline erinnern & kognitiv-mental \\
\hline empfinden & kognitiv-mental \\
\hline entwickeln & räumlich-haptisch \\
\hline eingehen auf & räumlich-haptisch \\
\hline legen & räumlich-haptisch \\
\hline betrachten & optisch-visuell \\
\hline glauben & kognitiv-mental \\
\hline richten & räumlich-haptisch \\
\hline auftreten & räumlich-haptisch \\
\hline äußern & akustisch-sprachlich \\
\hline beruhen auf & räumlich-haptisch \\
\hline erwähnen & akustisch-sprachlich \\
\hline treffen & räumlich-haptisch \\
\hline ausgehen von & räumlich-haptisch \\
\hline hinweisen & räumlich-haptisch \\
\hline beschränken & räumlich-haptisch \\
\hline aufnehmen & räumlich-haptisch \\
\hline erheben & räumlich-haptisch \\
\hline erfüllen & räumlich-haptisch \\
\hline erfahren & $\begin{array}{l}\text { räumlich-haptisch/ } \\
\text { kognitiv-mental }\end{array}$ \\
\hline lösen & räumlich-haptisch \\
\hline fassen & räumlich-haptisch \\
\hline begründen & räumlich-haptisch \\
\hline betonen & akustisch-sprachlich \\
\hline vertreten & räumlich-haptisch \\
\hline bewegen & räumlich-haptisch \\
\hline aussprechen & akustisch-sprachlich \\
\hline erzeugen & räumlich-haptisch \\
\hline anwenden & räumlich-haptisch \\
\hline einsetzen & räumlich-haptisch \\
\hline leiten & räumlich-haptisch \\
\hline untersuchen & $\begin{array}{l}\text { optisch-visuell/ } \\
\text { räumlich-haptisch }\end{array}$ \\
\hline trennen & räumlich-haptisch \\
\hline stammen & räumlich-haptisch \\
\hline beobachten & optisch-visuell \\
\hline liefern & räumlich-haptisch \\
\hline voraussetzen & räumlich-haptisch \\
\hline herstellen & räumlich-haptisch \\
\hline vorkommen & räumlich-haptisch \\
\hline
\end{tabular}

\begin{tabular}{|c|c|}
\hline begreifen & räumlich-haptisch \\
\hline lauten & akustisch-sprachlich \\
\hline ausdrücken & akustisch-sprachlich \\
\hline eintreten & räumlich-haptisch \\
\hline erfassen & räumlich-haptisch \\
\hline ausführen & räumlich-haptisch \\
\hline durchführen & räumlich-haptisch \\
\hline übernehmen & räumlich-haptisch \\
\hline wachsen & räumlich-haptisch \\
\hline umfassen & räumlich-haptisch \\
\hline ersetzen & räumlich-haptisch \\
\hline vorliegen & räumlich-haptisch \\
\hline fragen & akustisch-sprachlich \\
\hline hervorgehen & räumlich-haptisch \\
\hline kennzeichnen & räumlich-haptisch \\
\hline ausschließen & räumlich-haptisch \\
\hline aufgeben & räumlich-haptisch \\
\hline behaupten & akustisch-sprachlich \\
\hline festhalten & räumlich-haptisch \\
\hline auffassen & räumlich-haptisch \\
\hline greifen & räumlich-haptisch \\
\hline beherrschen & räumlich-haptisch \\
\hline erleben & kognitiv-mental \\
\hline vorstellen & räumlich-haptisch \\
\hline schildern & akustisch-sprachlich \\
\hline vornehmen & räumlich-haptisch \\
\hline lesen & akustisch-sprachlich \\
\hline verfolgen & räumlich-haptisch \\
\hline ablehnen & räumlich-haptisch \\
\hline durchsetzen & räumlich-haptisch \\
\hline vermitteln & räumlich-haptisch \\
\hline begegnen & räumlich-haptisch \\
\hline entdecken & $\begin{array}{l}\text { optisch-visuell/ } \\
\text { räumlich-haptisch }\end{array}$ \\
\hline malen & $\begin{array}{l}\text { optisch-visuell/ } \\
\text { räumlich-haptisch }\end{array}$ \\
\hline absehen & optisch-visuell \\
\hline orientieren & $\begin{array}{l}\text { räumlich-haptisch/ } \\
\text { optisch-visuell }\end{array}$ \\
\hline verbreiten & räumlich-haptisch \\
\hline anschließen & räumlich-haptisch \\
\hline eröffnen & räumlich-haptisch \\
\hline hervorheben & räumlich-haptisch \\
\hline
\end{tabular}




\begin{tabular}{|c|c|}
\hline bemerken & kognitiv-mental \\
\hline berichten & akustisch-sprachlich \\
\hline stützen & räumlich-haptisch \\
\hline erzählen & akustisch-sprachlich \\
\hline zusammenfassen & räumlich-haptisch \\
\hline zurückführen & räumlich-haptisch \\
\hline anlegen & räumlich-haptisch \\
\hline aufstellen & räumlich-haptisch \\
\hline gestalten & räumlich-haptisch \\
\hline beziehen & räumlich-haptisch \\
\hline abschließen & räumlich-haptisch \\
\hline aufbauen & räumlich-haptisch \\
\hline formulieren & akustisch-sprachlich \\
\hline übertragen & räumlich-haptisch \\
\hline begrenzen & räumlich-haptisch \\
\hline unterwerfen & räumlich-haptisch \\
\hline besagen & akustisch-sprachlich \\
\hline übergehen & räumlich-haptisch \\
\hline angehen & räumlich-haptisch \\
\hline antworten & akustisch-sprachlich \\
\hline prägen & räumlich-haptisch \\
\hline festlegen & räumlich-haptisch \\
\hline erörtern & räumlich-haptisch \\
\hline zuschreiben & $\begin{array}{l}\text { räumlich-haptisch/ } \\
\text { akustisch-sprachlich }\end{array}$ \\
\hline auflösen & räumlich-haptisch \\
\hline ausmachen & optisch-visuell \\
\hline erweitern & räumlich-haptisch \\
\hline berühren & räumlich-haptisch \\
\hline berücksichtigen & räumlich-haptisch \\
\hline entziehen & räumlich-haptisch \\
\hline interessieren & kognitiv-mental \\
\hline beachten & kognitiv-mental \\
\hline zurückkehren & räumlich-haptisch \\
\hline entgegensetzen & räumlich-haptisch \\
\hline widersprechen & akustisch-sprachlich \\
\hline neigen & räumlich-haptisch \\
\hline stoßen & räumlich-haptisch \\
\hline herausgeben & räumlich-haptisch \\
\hline wiedergeben & räumlich-haptisch \\
\hline anführen & räumlich-haptisch \\
\hline entfalten & räumlich-haptisch \\
\hline ansprechen & akustisch-sprachlich \\
\hline
\end{tabular}

\begin{tabular}{|c|c|}
\hline ausdehnen & räumlich-haptisch \\
\hline erschließen & räumlich-haptisch \\
\hline beitragen & räumlich-haptisch \\
\hline verknüpfen & räumlich-haptisch \\
\hline hervorrufen & $\begin{array}{l}\text { räumlich-haptisch/ } \\
\text { akustisch-sprachlich }\end{array}$ \\
\hline fortsetzen & räumlich-haptisch \\
\hline überwiegen & räumlich-haptisch \\
\hline wahrnehmen & $\begin{array}{l}\text { kognitiv-mental/ } \\
\text { räumlich-haptisch }\end{array}$ \\
\hline zuwenden & räumlich-haptisch \\
\hline sammeln & räumlich-haptisch \\
\hline gebären & räumlich-haptisch \\
\hline rücken & räumlich-haptisch \\
\hline hinzufügen & räumlich-haptisch \\
\hline begleiten & räumlich-haptisch \\
\hline einnehmen & räumlich-haptisch \\
\hline unterstützen & räumlich-haptisch \\
\hline nähern & räumlich-haptisch \\
\hline anpassen & räumlich-haptisch \\
\hline entnehmen & räumlich-haptisch \\
\hline entwerfen & räumlich-haptisch \\
\hline übersetzen & räumlich-haptisch \\
\hline bedienen & räumlich-haptisch \\
\hline decken & räumlich-haptisch \\
\hline belegen & räumlich-haptisch \\
\hline zusammensetzen & räumlich-haptisch \\
\hline entspringen & räumlich-haptisch \\
\hline einleiten & räumlich-haptisch \\
\hline unterliegen & räumlich-haptisch \\
\hline übersehen & optisch-visuell \\
\hline formen & räumlich-haptisch \\
\hline einschränken & räumlich-haptisch \\
\hline heranziehen & räumlich-haptisch \\
\hline umgeben & räumlich-haptisch \\
\hline zuordnen & räumlich-haptisch \\
\hline angreifen & räumlich-haptisch \\
\hline halten für & räumlich-haptisch \\
\hline beseitigen & räumlich-haptisch \\
\hline vorlegen & räumlich-haptisch \\
\hline darlegen & räumlich-haptisch \\
\hline betreiben & räumlich-haptisch \\
\hline feststehen & räumlich-haptisch \\
\hline
\end{tabular}




\begin{tabular}{|c|c|}
\hline einbeziehen & räumlich-haptisch \\
\hline herbeiführen & räumlich-haptisch \\
\hline überwinden & räumlich-haptisch \\
\hline durchdringen & räumlich-haptisch \\
\hline zurückgehen auf & räumlich-haptisch \\
\hline anknüpfen & räumlich-haptisch \\
\hline zusammenstellen & räumlich-haptisch \\
\hline anbieten & räumlich-haptisch \\
\hline herausstellen & räumlich-haptisch \\
\hline verkörpern & räumlich-haptisch \\
\hline unterordnen & räumlich-haptisch \\
\hline verwerfen & räumlich-haptisch \\
\hline zusammenfallen & räumlich-haptisch \\
\hline vorfinden & räumlich-haptisch \\
\hline unterstellen & räumlich-haptisch \\
\hline fortschreiten & räumlich-haptisch \\
\hline zugeben & räumlich-haptisch \\
\hline ausbreiten & räumlich-haptisch \\
\hline vertiefen & räumlich-haptisch \\
\hline konzentrieren & kognitiv-mental \\
\hline befassen & räumlich-haptisch \\
\hline enthüllen & räumlich-haptisch \\
\hline abweichen & räumlich-haptisch \\
\hline klären & optisch-visuell \\
\hline einsehen & optisch-visuell \\
\hline beleuchten & optisch-visuell \\
\hline verschmelzen & räumlich-haptisch \\
\hline präsentieren & $\begin{array}{l}\text { optisch-visuell/ } \\
\text { räumlich-haptisch }\end{array}$ \\
\hline verdrängen & räumlich-haptisch \\
\hline gegenüberstellen & räumlich-haptisch \\
\hline fixieren & räumlich-haptisch \\
\hline gliedern & räumlich-haptisch \\
\hline bedenken & kognitiv-mental \\
\hline verteidigen & räumlich-haptisch \\
\hline spiegeln & optisch-visuell \\
\hline abgrenzen & räumlich-haptisch \\
\hline einwirken & räumlich-haptisch \\
\hline ansetzen & räumlich-haptisch \\
\hline hinausgehen & räumlich-haptisch \\
\hline herausarbeiten & räumlich-haptisch \\
\hline vorsehen & optisch-visuell \\
\hline aussehen & optisch-visuell \\
\hline
\end{tabular}

\begin{tabular}{|l|l|}
\hline aufgreifen & räumlich-haptisch \\
\hline konstruieren & räumlich-haptisch \\
\hline umsetzen & räumlich-haptisch \\
\hline
\end{tabular}

\begin{tabular}{|c|c|}
\hline Nomen & Bildfeld \\
\hline Frage & optisch-visuell \\
\hline Grund & räumlich-haptisch \\
\hline Arbeit & räumlich-haptisch \\
\hline Verhältnis & räumlich-haptisch \\
\hline Beziehung & räumlich-haptisch \\
\hline Zusammenhang & räumlich-haptisch \\
\hline Bewegung & räumlich-haptisch \\
\hline Gebiet & räumlich-haptisch \\
\hline Ausdruck & $\begin{array}{l}\text { räumlich-haptisch/ } \\
\text { akustisch-sprachlich }\end{array}$ \\
\hline Darstellung & räumlich-haptisch \\
\hline Denken & kognitiv-mental \\
\hline Einfluss & räumlich-haptisch \\
\hline Raum & räumlich-haptisch \\
\hline Interesse & kognitiv-mental \\
\hline Bewusstsein & kognitiv-mental \\
\hline Vorstellung & kognitiv-mental \\
\hline Gegenstand & räumlich-haptisch \\
\hline Gedanke & kognitiv-mental \\
\hline Erkenntnis & kognitiv-mental \\
\hline Verbindung & räumlich-haptisch \\
\hline Erfahrung & kognitiv-mental \\
\hline Richtung & räumlich-haptisch \\
\hline Auffassung & $\begin{array}{l}\text { kognitiv-mental/ } \\
\text { räumlich-haptisch }\end{array}$ \\
\hline Grundlage & räumlich-haptisch \\
\hline Grenze & räumlich-haptisch \\
\hline Stellungnahme & räumlich-haptisch \\
\hline Schule & räumlich-haptisch \\
\hline Strömung & räumlich-haptisch \\
\hline Punkt & räumlich-haptisch \\
\hline Bereich & räumlich-haptisch \\
\hline Quelle & räumlich-haptisch \\
\hline Rede & akustisch-sprachlich \\
\hline Glaube & kognitiv-mental \\
\hline Linie & räumlich-haptisch \\
\hline Wissen & kognitiv-mental \\
\hline Zeichen & optisch-visuell \\
\hline
\end{tabular}




\begin{tabular}{|c|c|}
\hline Blick & optisch-visuell \\
\hline Betrachtung & optisch-visuell \\
\hline Anschauung & optisch-visuell \\
\hline Blickwinkel & $\begin{array}{l}\text { räumlich-haptisch/ } \\
\text { optisch-visuell }\end{array}$ \\
\hline Rahmen & räumlich-haptisch \\
\hline Beitrag & räumlich-haptisch \\
\hline Annahme & $\begin{array}{l}\text { kognitiv-mental/ } \\
\text { räumlich-haptisch }\end{array}$ \\
\hline Schritt & räumlich-haptisch \\
\hline Gesichtspunkte & räumlich-haptisch \\
\hline Beobachtung & optisch-visuell \\
\hline Eindruck & $\begin{array}{l}\text { kognitiv-mental/ } \\
\text { räumlich-haptisch }\end{array}$ \\
\hline Bezug & räumlich-haptisch \\
\hline Verständnis & kognitiv-mental \\
\hline Einblick & optisch-visuell \\
\hline Hinsicht & optisch-visuell \\
\hline Ansicht & optisch-visuell \\
\hline Ausführung & räumlich-haptisch \\
\hline Übergang & räumlich-haptisch \\
\hline Ebene & räumlich-haptisch \\
\hline Ansatz & räumlich-haptisch \\
\hline Verlauf & räumlich-haptisch \\
\hline Landschaft & räumlich-haptisch \\
\hline Spannung & räumlich-haptisch \\
\hline Abhängigkeit & räumlich-haptisch \\
\hline Gegensatz & räumlich-haptisch \\
\hline Überlieferung & räumlich-haptisch \\
\hline Deutung & $\begin{array}{l}\text { kognitiv-mental/ } \\
\text { räumlich-haptisch }\end{array}$ \\
\hline Hinweis & räumlich-haptisch \\
\hline Neigung & räumlich-haptisch \\
\hline Umfang & räumlich-haptisch \\
\hline Gang & räumlich-haptisch \\
\hline Einsicht & optisch-visuell \\
\hline Ursprung & räumlich-haptisch \\
\hline Fortschritt & räumlich-haptisch \\
\hline Einstellung & $\begin{array}{l}\text { kognitiv-mental/ } \\
\text { räumlich-haptisch }\end{array}$ \\
\hline Wahrnehmung & kognitiv-mental \\
\hline Widerstand & räumlich-haptisch \\
\hline Zweifel & kognitiv-mental \\
\hline
\end{tabular}

\begin{tabular}{|l|l|}
\hline Konflikt & räumlich-haptisch \\
\hline Hintergrund & räumlich-haptisch \\
\hline Vordergrund & räumlich-haptisch \\
\hline Gewicht & räumlich-haptisch \\
\hline Aufmerksamkeit & kognitiv-mental \\
\hline Fokus & optisch-visuell \\
\hline Feststellung & räumlich-haptisch \\
\hline Diskussion & akustisch-sprachlich \\
\hline Mittelpunkt & räumlich-haptisch \\
\hline Verstehen & kognitiv-mental \\
\hline Oberfläche & räumlich-haptisch \\
\hline Entdeckung & räumlich-haptisch \\
\hline Übereinstimmung & räumlich-haptisch \\
\hline Abstand & räumlich-haptisch \\
\hline Einführung & räumlich-haptisch \\
\hline Einleitung & räumlich-haptisch \\
\hline Ausblick & optisch-visuell \\
\hline Tiefe & räumlich-haptisch \\
\hline Nähe & räumlich-haptisch \\
\hline Position & räumlich-haptisch \\
\hline Basis & räumlich-haptisch \\
\hline &
\end{tabular}

\begin{tabular}{|l|l|}
\hline Adjektiv & Bildfeld \\
\hline groß & räumlich-haptisch \\
\hline weit & räumlich-haptisch \\
\hline hoch & räumlich-haptisch \\
\hline stark & räumlich-haptisch \\
\hline klein & räumlich-haptisch \\
\hline nahe & räumlich-haptisch \\
\hline rein & optisch-visuell \\
\hline folgend & räumlich-haptisch \\
\hline kurz & räumlich-haptisch \\
\hline knapp & räumlich-haptisch \\
\hline schwer & räumlich-haptisch \\
\hline tief & räumlich-haptisch \\
\hline ursprünglich & räumlich-haptisch \\
\hline klar & optisch-visuell \\
\hline eng & räumlich-haptisch \\
\hline fern & räumlich-haptisch \\
\hline fest & räumlich-haptisch \\
\hline streng & kognitiv-mental \\
\hline scharf & räumlich-haptisch \\
\hline unabhängig & räumlich-haptisch \\
\hline &
\end{tabular}




\begin{tabular}{|c|c|}
\hline offen & räumlich-haptisch \\
\hline fremd & kognitiv-mental \\
\hline erheblich & räumlich-haptisch \\
\hline grundsätzlich & räumlich-haptisch \\
\hline grundlegend & räumlich-haptisch \\
\hline gegenwärtig & räumlich-haptisch \\
\hline breit & räumlich-haptisch \\
\hline erschöpfend & räumlich-haptisch \\
\hline umfassend & räumlich-haptisch \\
\hline weitgehend & räumlich-haptisch \\
\hline sichtbar & optisch-visuell \\
\hline zentral & räumlich-haptisch \\
\hline unbekannt & kognitiv-mental \\
\hline außerordentlich & räumlich-haptisch \\
\hline geschlossen & räumlich-haptisch \\
\hline selbstverständlich & kognitiv-mental \\
\hline abhängig & räumlich-haptisch \\
\hline mittlere & räumlich-haptisch \\
\hline genannt & akustisch-sprachlich \\
\hline stehend & räumlich-haptisch \\
\hline ausdrücklich & akustisch-sprachlich \\
\hline ausführlich & räumlich-haptisch \\
\hline schwach & räumlich-haptisch \\
\hline niedrig & räumlich-haptisch \\
\hline eingehend & räumlich-haptisch \\
\hline scheinbar & optisch-visuell \\
\hline zugänglich & räumlich-haptisch \\
\hline vorwiegend & räumlich-haptisch \\
\hline entwickelt & räumlich-haptisch \\
\hline parallel & räumlich-haptisch \\
\hline umfangreich & räumlich-haptisch \\
\hline ausgehend & räumlich-haptisch \\
\hline vorliegend & räumlich-haptisch \\
\hline anschaulich & optisch-visuell \\
\hline anschließend & räumlich-haptisch \\
\hline folglich & räumlich-haptisch \\
\hline entfernt & räumlich-haptisch \\
\hline merkwürdig & kognitiv-mental \\
\hline offensichtlich & optisch-visuell \\
\hline begrenzt & räumlich-haptisch \\
\hline begrifflich & räumlich-haptisch \\
\hline hervorragend & räumlich-haptisch \\
\hline beschränkt & räumlich-haptisch \\
\hline
\end{tabular}

\begin{tabular}{|l|l|}
\hline überwiegend & räumlich-haptisch \\
\hline ausgeprägt & räumlich-haptisch \\
\hline denkbar & kognitiv-mental \\
\hline ausgesprochen & akustisch-sprachlich \\
\hline entgegengesetzt & räumlich-haptisch \\
\hline überliefert & räumlich-haptisch \\
\hline benachbart & räumlich-haptisch \\
\hline verwandt & räumlich-haptisch \\
\hline starr & räumlich-haptisch \\
\hline fließend & räumlich-haptisch \\
\hline orientiert & räumlich-haptisch/ \\
& optisch-visuell \\
\hline flach & räumlich-haptisch \\
\hline grob & räumlich-haptisch \\
\hline fein & räumlich-haptisch \\
\hline überflüssig & räumlich-haptisch \\
\hline fortschreitend & räumlich-haptisch \\
\hline auffallend & räumlich-haptisch \\
\hline ausgedehnt & räumlich-haptisch \\
\hline zusammenhängend & räumlich-haptisch \\
\hline treffend & räumlich-haptisch \\
\hline trocken & räumlich-haptisch \\
\hline leitend & räumlich-haptisch \\
\hline nachfolgend & räumlich-haptisch \\
\hline plastisch & räumlich-haptisch \\
\hline vielseitig & räumlich-haptisch \\
\hline stammend & räumlich-haptisch \\
\hline angelegt & räumlich-haptisch \\
\hline &
\end{tabular}

